\title{
Theoretical modeling of crystalline symmetry order with dendritic morphology
}

\author{
L.V. Toropova ${ }^{1, a}$, P.K. Galenko ${ }^{2}$, D.V. Alexandrov ${ }^{1}$, G. Demange ${ }^{3}$, A. Kao $^{4}$, and \\ M. Rettenmayr ${ }^{2}$ \\ 1 Department of Theoretical and Mathematical Physics, Laboratory of Multi-Scale Mathe- \\ matical Modeling, Ural Federal University, Lenin Ave., 51, Ekaterinburg, 620000, Russian \\ Federation \\ ${ }^{2}$ Friedrich-Schiller-Universität-Jena, Physikalisch-Astronomische Fakultät, 07743 Jena, Ger- \\ many \\ 3 GPM, CNRS-UMR 6634, University of Rouen Normandy, 76801, Saint Ètienne Du Rou- \\ vray, France \\ 4 Centre for Numerical Modelling and Process Analysis, University of Greenwich, Old Royal \\ Naval College, Park Row, London SE10 9LS, UK
}

\begin{abstract}
The stable growth of a crystal with dendritic morphology with $n$-fold symmetry is modeled. Using the linear stability analysis and solvability theory, a selection criterion for thermally and solutally controlled growth of the dendrite is derived. A complete set of nonlinear equations consisting of the selection criterion and an undercooling balance (which determines the implicit dependencies of the dendrite tip velocity and tip diameter on the total undercooling) is formulated. The growth kinetics of crystals having different lattice symmetry is analyzed. The model predictions are compared with phase field modeling data on ice dendrites grown from pure undercooled water.
\end{abstract}

\section{Introduction}

A complex tree-like structure containing the main stem and side branches is called a dendrite. Such structures arise in different phase and structural transformation processes, ranging from the growth of individual crystals in a metastable system in materials science, to the domain evolution in ferroelectrics, crystallization of lava lakes, sea ice and at the inner core boundary of the Earth in geophysics to biology, chemical industry and life science $[1,2]$.

Theoretical advance and experimental verification of dendritic models were mainly achieved for crystals having a given (for instance, four-fold) symmetry. To date, there are no comparative analyses of dendrite growth exhibiting a full spectrum of crystalline symmetries. Therefore, the purpose of this paper is to evaluate the growth kinetics of crystals with a variety of lattice symmetries. The model predictions are compared with the growth kinetics of ice crystals growing from undercooled water (see Figure 1).

a e-mail: 1.v.toropova@urfu.ru 
(a)

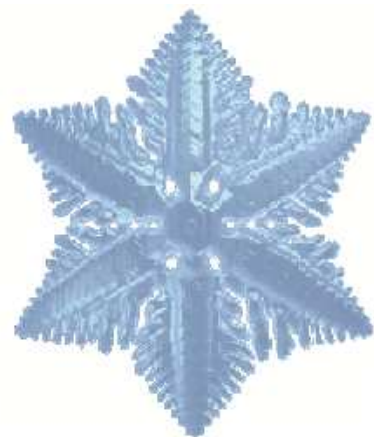

(b)

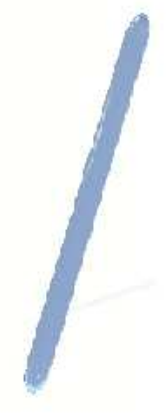

Fig. 1. Phase field simulation of ice dendrites, using the software Blender for visual rendering $[4,5]$. (a) Free-growing dendrite with six-fold symmetry $(n=6)$. (b) Column with two-fold symmetry $(n=2)$.

Crystal growth kinetics was modeled by level set method, phase field method, cellular automata, and sharp interface approaches [3]. For example, phase field simulations have been performed on the supercomputer Myria of Normandy (France) [4, 5]. Such devices allow massive parallel computing, which provides necessary computational power, by means of memory and calculation distribution between cores. In such simulations $[4,5]$, modeling of ice dendrites has been performed using the parallel FFTW routine for parallel Fourier transform of linear terms in the phase field equations.

Another well-established method is the enthalpy method $[6,7]$. In this method a single energy conservation equation for the entire solution domain, solid and liquid, is written in terms of the enthalpy - the sum of sensible and latent heats. The solution can be made on a fixed space grid and the interface tracked by use of the liquid fraction $0 \leq f \leq 1$, which plays a role of a parameter that retains the feature of an order parameter. The enthalpy method is successful at solving basic solidification problems where no undercoolings are presented and the equilibrium solidification temperature is a fixed constant. Here we present results on dendritic solidification of undercooled stagnant and flowing liquids due to the fact that the enthalpy method can be combined with lattice Boltzmann methods to simulate dendritic growth of a pure material in the presence of a flow field [8].

In the present work, we use a sharp interface approach for the detailed prediction of velocity $V$ and tip diameter $\rho$ of dendrites growing with different symmetry. With this aim, we summarize the obtained results on solvability analysis within the framework of the sharp interface model and discuss some theoretical predictions in terms of dendrite velocity $V$ and tip diameter $\rho$ as functions of undercooling $\Delta T$. The main parameters for dendritic growth $V$ and $\rho$ are compared with experimental data on the crystallization of snow crystals, using a phase field model.

\section{Crystal growth model}

The present system of equations is based on the advancements published in Refs. [911]. The convective heat and mass transport equations in the solid and liquid phases take the form

$$
\frac{\partial T_{l}}{\partial t}+(\mathbf{w} \cdot \nabla) T_{l}=D_{T} \nabla^{2} T_{l}, \quad \frac{\partial T_{s}}{\partial t}=D_{T} \nabla^{2} T_{s}
$$




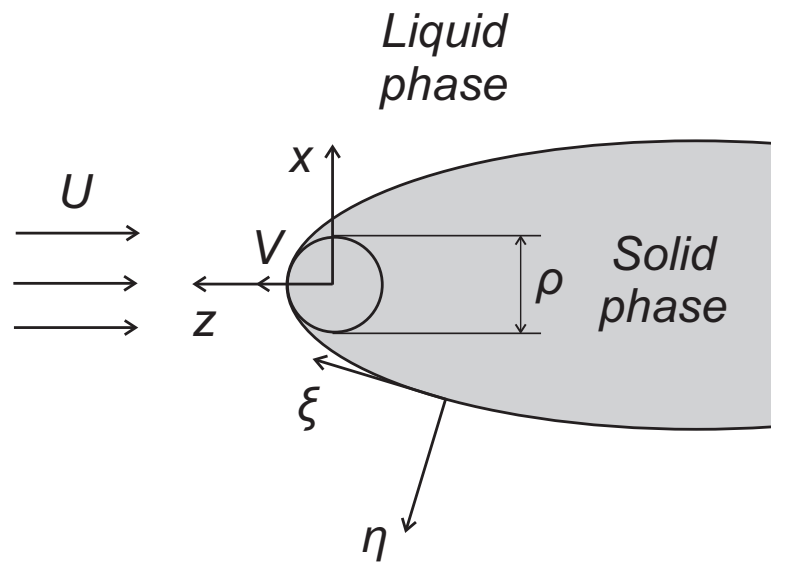

Fig. 2. A sketch of a dendrite tip in the presence of forced liquid flow. $U$ is the fluid flow velocity, $V$ is the dendrite growth velocity, $\rho$ is the dendrite tip diameter, $(x, z)$ and $(\xi, \eta)$ are the Cartesian and the parabolic coordinate systems, respectively.

$$
\frac{\partial C_{l}}{\partial t}+(\mathbf{w} \cdot \nabla) C_{l}=D_{C} \nabla^{2} C_{l}
$$

where $T_{l}$ and $T_{S}$ are the temperatures in liquid and solid respectively, $D_{T}$ is the thermal diffusivity, $D_{C}$ is the chemical diffusion coefficient, $C_{l}$ is the solute concentration in the liquid phase and $\mathbf{w}$ is the fluid velocity.

Here we traditionally neglect the atomic diffusion in the solid. For the sake of simplicity, we consider the case of equal thermal diffusivities in both phases. This hypothesis does not change the selection criteria given below, due to the fact that different thermal diffusivities will change the selection constant only.

To describe the hydrodynamic flows we use the linearized Oseen model for a viscous flow $[12-14]$

$$
U \frac{\partial \mathbf{w}}{\partial z}=-\frac{1}{\rho_{l}} \nabla p+\nu \nabla^{2} \mathbf{w}, \quad \nabla \cdot \mathbf{w}=0 .
$$

Here $U$ is the fluid flow velocity far from the dendrite surface (see Figure 2), $p$ is the pressure, $\rho_{l}$ is the density of the liquid, and $\nu$ is the kinematic viscosity.

The heat and mass balance conditions are fulfilled at the solid/liquid interface

$$
\begin{gathered}
T_{Q}(\mathbf{v} \cdot \mathbf{n})=D_{T}\left(\nabla T_{s}-\nabla T_{l}\right) \cdot \mathbf{n}, \\
\left(1-k_{0}\right) C_{l}(\mathbf{v} \cdot \mathbf{n})+D_{C} \nabla C_{l} \cdot \mathbf{n}=0,
\end{gathered}
$$

where $T_{Q}=Q / c_{p}$ is the hypercooling temperature (temperature for complete adiabatic solidification), $(\mathbf{v} \cdot \mathbf{n})=v_{n}$ is the normal growth velocity, where $\mathbf{n}$ is the unit normal to the interface, $k_{0}$ represents the equilibrium partition coefficient.

The Gibbs-Thomson equation is applied at the solid/liquid boundary holds,

$$
T_{\mathrm{int}}=T_{l}=T_{s}=T_{m}-m C_{l}-T_{Q} d(\theta, \phi) \mathcal{K}-\tilde{\beta}(\theta, \phi) v_{n},
$$

where $m$ is the liquidus slope. The curvature is given by

$$
\mathcal{K}=\left\{\begin{array}{l}
1 / R, \quad \text { two }- \text { dimensional } \quad(2 \mathrm{D}) \text { space, } \\
\left(R_{1}+R_{2}\right) /\left(R_{1} R_{2}\right), \text { three }- \text { dimensional }(3 \mathrm{D}) \text { space },
\end{array}\right.
$$


where $R$ is the dendrite tip radius in $2 \mathrm{D}$ and $R_{1}$ and $R_{2}$ are the main radii of curvature for $3 \mathrm{D}$ dendrite tip.

At the dendrite interface the phase transition temperature $T_{\text {int }}$ in Eq. (6) is determined by the specific heat $c_{p}$, the latent heat of solidification $Q$, the anisotropic capillary length $d(\theta, \phi)$, the interface curvature $\mathcal{K}$, the melting temperature $T_{m}$ for the pure solvent and the intensity of the atomic kinetics. This entity is given by the function of anisotropic kinetics $\tilde{\beta}(\theta, \phi)$ with the spherical angles $\theta$ and $\phi$, which define the orientation between the normal to the dendrite surface and its growth direction.

In the case of a needle-like crystal for obtaining the selection criterion $\sigma^{*}$ one can neglect changes along $\phi$ angle [15] and use the simplest form of anisotropy under the assumption that final scaling may be applied to 3D dendrite growth, in which $d(\theta)$ and $\tilde{\beta}(\theta)$ are written for the case of $n$-fold symmetry:

$$
d(\theta)=d_{0}\left\{1-\alpha_{d} \cos \left[n\left(\theta-\theta_{d}\right)\right]\right\}, \quad \tilde{\beta}(\theta)=\beta_{0} T_{Q}\left\{1-\alpha_{\beta} \cos \left[n\left(\theta-\theta_{\beta}\right)\right]\right\},
$$

where $d_{0}$ and $\beta_{0}$ are the capillary and kinetic constants respectively, $\alpha_{d}$ and $\alpha_{\beta}$ are the small anisotropy parameters, so that $\alpha_{d} \ll 1$ and $\alpha_{\beta} \ll 1, \theta_{d}$ and $\theta_{\beta}$ designate the angles between the directions of growth and the minimal functions $d(\theta)$ and $\tilde{\beta}(\theta)$, $n$ is the lattice symmetry order, which influences on the crystal growth kinetics.

The far field conditions are written in the form

$$
C_{l}=C_{0}, \quad T_{l}=T_{\infty}, \quad|\mathbf{w}|=U
$$

\section{Selection of stable mode}

In the case of low surface energy and anisotropy of kinetics, the temperature and solute concentration distributions are in close proximity to the parabolic Ivantsov solutions describing steady-state dendritic growth. Pelcé and Bensimon [16] (see also [17, 18]) showed that this statement leads to the microscopic solvability condition which represents an approximate analytical solution of the linearized heat and mass transfer equations at the parabolic surface of an Ivantsov dendrite. As shown below, this solvability condition gives a unique combination of $\rho$ and $V$ and describes a stable dendritic growth mode. After Pelcé and Bensimon [16] the solvability condition is given by

$$
\int_{-\infty}^{\infty} G\left[X_{0}(l)\right] Y_{m}(l) d l=0, \quad Y_{m}(l)=\exp \left[i \int_{0}^{l} k_{m}\left(l_{1}\right) d l_{1}\right],
$$

where $G$ designates the curvature operator, $k_{m}(l)$ the marginal wavenumber mode, $i$ an imaginary unit, and $X_{0}(l)$ a continuum of solutions from which the dependence $k_{m}(l)$ can be derived. Expression (10) describes different shapes of evolving phase interfaces [17] and has the following meaning: the solution $G\left[X_{0}(l)\right]$ is orthogonal to the imposed perturbation $Y_{m}(l)$ that provides a stable mode. This condition follows from the Wentzel-Kramers-Brillouin method [19] which was also applied for the propagation of a flame [20] and the interfaces of needle-like crystals [21].

To obtain the marginal wavenumbers $k_{m}$ entering in the solvability integral (10) we carry out a linear stability analysis [11]. In this case, the marginal mode of the wavenumber $k_{m}$ (see $[18,22]$ for details), can be determined by the cubic equation

$$
\begin{array}{r}
k_{m}^{3}=\frac{V \exp (i \theta)}{2 d(\theta) D_{T}} k_{m}+\frac{i a U \sin \theta \cos \theta}{8 \rho D_{T}} k_{m} \\
-\frac{i V \sin \theta}{2 D_{T}} k_{m}^{2}+\frac{V^{2} \cos \theta \exp (i \theta)}{4 d(\theta) D_{T}^{2}}+\frac{i V \tilde{\beta}(\theta) \sin \theta}{d(\theta) T_{Q}} k_{m}^{2},
\end{array}
$$


where

$$
a=\frac{\exp (-\Re / 2)}{E_{1}(\Re / 2)}, \quad \Re=\frac{\rho U}{\nu}, \quad E_{1}(q)=\int_{q}^{\infty} \frac{\exp (-u)}{u} d u
$$

and $V=v_{n}$ at the dendrite tip.

Substituting the analytical solution of the cubic equation (11) into the solvability integral (10), one can obtain a solvability criterion. We calculate the solvability integral (10) in two stages, as detailed in [9]. First, we neglect the kinetic contribution (proportional to $\beta_{0}$ ) and only pay attention to the "thermally controlled" case of crystal growth. Setting $\beta_{0}=0$, we come to the selection criterion (see also $[10,18$, 22])

$$
\begin{gathered}
\sigma^{*}=\frac{2 d_{0} D_{T}}{\rho^{2} V}=\frac{\sigma_{0} \alpha_{d}^{7 / n} A_{n}^{7 / n}}{\left(1+a_{1} \alpha_{d}^{2 / n} A_{n}^{2 / n} P_{g}\right)^{2}\left(1+b \tau_{n}^{\gamma_{n}}\right)}, \\
\tau_{n}=\alpha \alpha_{d}^{-3 / n} A_{n}^{-3 / n}, \quad \alpha=\frac{a U d_{0}}{4 \rho V}, \quad \Upsilon_{n}=\frac{n+7}{2(n+3)},
\end{gathered}
$$

where

$$
A_{n}=2^{-3 n / 4} \sum_{k=0}^{n}\left(\begin{array}{l}
n \\
k
\end{array}\right) i^{n-k} \cos \frac{(n-k) \pi}{2}, \quad\left(\begin{array}{l}
n \\
k
\end{array}\right)=C_{n}^{k} \frac{n !}{k !(n-k) !},
$$

and $\sigma_{0}, a_{1}$ and $b$ are the constants, $C_{n}^{k}$ is the number of $k$-combinations from a given set of $n$ elements. It is important that the order of symmetry $n$ in eq. (12) cannot be arbitrary. It is limited by the crystal symmetry $(\mathrm{n}=2,3,4,6)$ or quasi-crystal symmetry $(\mathrm{n}=10)$. For the given values of $n$, the parameter $A_{n}$ is always a real number, see Table 1 .

Table 1. Parameters from the selection criterion (13) in a 2D geometry

\begin{tabular}{lll}
\hline \hline Symmetry order $n$ & Crystalline structure & Parameter $A_{n}$ \\
\hline 10 & quasicrystals & $2^{3 / 2}$ \\
6 & snowflakes & $2^{1 / 2}$ \\
4 & squares/rectangles & 1 \\
3 & triangles & $2^{-1 / 4}$ \\
2 & whiskers & $2^{-1 / 2}$ \\
\hline
\end{tabular}

In the second stage of evaluating the integral expression (10) we analyze the dendrite growth mode that is controlled by the kinetic contribution which in turn is proportional to $\beta_{0}$ (see eq. (3.16) in [11]). By doing so, we arrive at (by analogy with the analysis, presented in the work [9])

$$
\sigma^{*}=\frac{\sigma_{0} \alpha_{d}^{7 / n} A_{n}^{7 / n}}{\left[1+a_{1}^{\prime} \alpha_{d}^{2 / n} P_{g} D_{T} \beta_{0} A_{n}^{2 / n} / d_{0}\right]^{2}\left(1+b \tau_{n}^{\Upsilon_{n}}\right)} .
$$

Sewing together the obtained limiting criteria (12) and (14), we come to a generalized selection criterion in the form

$$
\sigma^{*}=\frac{\sigma_{0} \alpha_{d}^{7 / n} A_{n}^{7 / n}}{\left[1+a_{1} \alpha_{d}^{2 / n} A_{n}^{2 / n} P_{g}\left(1+\delta_{0} D_{T} \beta_{0} / d_{0}\right)\right]^{2}\left(1+b \tau_{n}^{\Upsilon_{n}}\right)},
$$


where $a_{1}^{\prime}=a_{1} \delta_{0}$ and $\delta_{0}$ represents the constant which is sewing the diffusioncontrolled and kinetically-limited regimes of dendrite growth [9]. Note that the selection constants $\sigma_{0}$ and $b$ for each $n$ can be found experimentally, or from the phase-field modeling.

Now consider a dendrite of a binary alloy growing in the presence of convection at a constant temperature. The problem statement and solvability analysis are quite similar to the aforementioned purely thermal dendrites. The difference lies in the fact that the alloy dendrite is described by a one-sided model (diffusion in the liquid controls growth, and the diffusion in the solid is negligible). This gives a scale factor of " 2 " in the solvability criterion. In addition, keeping in mind that a correlation multiplier $m C_{i}\left(1-k_{0}\right) / T_{Q}$ appears as a result of the transition from a purely thermal to purely solutal Stefan problem, we come to a stability criterion $\sigma_{\mathrm{CD}}^{*}$ that describes stable dendritic growth in a two-component isothermal system with forced convective flow as

$$
\sigma_{\mathrm{CD}}^{*}=\frac{2 d_{0} D_{C}}{\rho^{2} V}=\frac{\left[2 m C_{i}\left(1-k_{0}\right) / T_{Q}\right] \sigma_{0} \alpha_{d}^{7 / n} A_{n}^{7 / n}}{\left[1+a_{2} \alpha_{d}^{2 / n} A_{n}^{2 / n} P_{C}\left(1+\delta_{0} D_{C} \beta_{0} / d_{0 \mathrm{CD}}\right)\right]^{2}\left(1+b \tau_{n \mathrm{CD}}^{\Upsilon_{n}}\right)},
$$

where

$$
\tau_{n \mathrm{CD}}=\frac{\alpha_{\mathrm{CD}}}{\alpha_{d}^{3 / n} A_{n}^{3 / n}}, \quad \alpha_{\mathrm{CD}}=\frac{a U d_{0 \mathrm{CD}}}{2 \rho V}, \quad d_{0 \mathrm{CD}}=\frac{T_{Q} d_{0}}{2 m C_{i}\left(1-k_{0}\right)}, \quad P_{C}=\frac{\rho V}{2 D_{C}},
$$

$k_{0}$ represents the equilibrium partition coefficient and $a_{2}=\sqrt{2} a_{1}$ [10].

The two selection criteria (15) and (16), derived for thermal and chemically stable dendritic growth, are joined to a unified criterion for the thermo-solutal problem. The unified selection criterion which gives a combination of $V$ and $\rho$ for dendritic growth in binary systems with forced convection and which takes into account the anisotropies of growth kinetics and surface energy reads as

$$
\begin{aligned}
\sigma^{*}=\frac{2 d_{0} D_{T}}{\rho^{2} V}= & \frac{\sigma_{0} \alpha_{d}^{7 / n} A_{n}^{7 / n}}{1+b \bar{\tau}_{n}^{\gamma_{n}}}\left\{\frac{1}{\left[1+a_{1} \alpha_{d}^{2 / n} A_{n}^{2 / n} P_{g}\left(1+\delta_{0} D_{T} \beta_{0} / d_{0}\right)\right]^{2}}\right. \\
& \left.+\frac{2 m C_{i}\left(1-k_{0}\right) D_{T}}{\left[1+a_{2} \alpha_{d}^{2 / n} A_{n}^{2 / n} P_{C}\left(1+\delta_{0} D_{C} \beta_{0} / d_{0 \mathrm{CD}}\right)\right]^{2} T_{Q} D_{C}}\right\}
\end{aligned}
$$

where

$$
\bar{\tau}_{n}=\alpha_{d}^{-3 / n} A_{n}^{-3 / n}\left(\frac{a U d_{0}}{4 \rho V P}+\frac{a U d_{0} D_{T}}{2 \rho V P D_{C}}\right), \quad P=1+\frac{2 m C_{i}\left(1-k_{0}\right) D_{T}}{T_{Q} D_{C}} .
$$

The criterion (17) works for arbitrary Péclet numbers within the framework of the parabolic heat and mass transfer model. Note that the generalized selection criterion for binary melts taking into account the $n$-fold symmetry of crystalline anisotropy, arbitrary Péclet numbers (including the rapid crystallization scenario) and a forced convection was recently derived by Alexandrov and Galenko in [23].

\section{Undercooling balance}

The selection criterion (17) obtained for a $n$-fold symmetry determines the first combination of crystal tip velocity $V$ and tip diameter $\rho$ as $\sigma^{*}=2 d_{0} D_{T} /\left(\rho^{2} V\right)$. This 
combination is the result of solutions close to the steady-state Ivantsov or HorvayCahn solutions which determine the temperature and concentration at the surface of dendrites.

The second combination of $V$ and $\rho$ is found from the undercooling balance which represents the driving force for crystal growth. The total undercooling $\Delta T=T_{l}-T_{\infty}$ at the dendrite tip consists of several contributions

$$
\Delta T=\Delta T_{T}+\Delta T_{C}+\Delta T_{R}+\Delta T_{K}
$$

Here $\Delta T_{T}$ and $\Delta T_{C}$ are the thermal and solutal undercoolings which can be written in a form

$$
\Delta T_{T}=T_{Q} \operatorname{Iv}_{T}^{*}\left(P_{g}, P_{f}\right), \quad \Delta T_{C}=\frac{m C_{\infty}\left(1-k_{0}\right) \operatorname{Iv}_{C}^{*}\left(P_{C}, P_{c f}\right)}{1-\left(1-k_{0}\right) \operatorname{Iv}_{C}^{*}\left(P_{C}, P_{c f}\right)},
$$

where the modified thermal and solutal Ivantsov functions are given by

$$
\begin{gathered}
\operatorname{Iv}_{T}^{*}\left(P_{g}, P_{f}\right)=P_{g} \exp \left(P_{0}\right) I_{T}(\infty), \quad P_{0}=P_{g}+P_{f}, \\
\operatorname{Iv}_{C}^{*}\left(P_{C}, P_{c f}\right)=P_{C} \exp \left(P_{C}+P_{c f}\right) I_{C}(\infty), \\
I_{T}(\infty)=\int_{1}^{\infty} \exp \left[(j-1) P_{f} \int_{1}^{\eta^{\prime}} \frac{g\left(\eta^{\prime \prime}\right)}{\sqrt{\eta^{\prime \prime}}} d \eta^{\prime \prime}-P_{0} \eta^{\prime}\right] \frac{d \eta^{\prime}}{\eta^{\prime(j-1) / 2}}, \\
I_{C}(\infty)=\int_{1}^{\infty} \exp \left[(j-1) P_{f} \frac{D_{T}}{D_{C}} \int_{1}^{\eta^{\prime}} \frac{g\left(\eta^{\prime \prime}\right)}{\sqrt{\eta^{\prime \prime}}} d \eta^{\prime \prime}-P_{0} \frac{D_{T}}{D_{C}} \eta^{\prime}\right] \frac{d \eta^{\prime}}{\eta^{\prime(j-1) / 2}}
\end{gathered}
$$

and $j=3$ in a three-dimensional geometry.

$\Delta T_{R}=2 d_{0} T_{Q} / R$ is the undercooling due to the Gibbs-Thomson effect, and $\Delta T_{K}=V / \mu_{k}$ is the kinetic undercooling (where $\mu_{k}$ is the kinetic coefficient, which is related to $\beta_{0}$ from equation (8) as $\left.\left(\mu_{k} \cdot T_{Q}\right)^{-1}=\beta_{0}\right)$. The total undercooling balance $(18)$ connects the surface temperature $T_{i}$ at the dendrite tip and the far-field temperature $T_{\infty}$.

In order to find the stable mode of crystal growth in a binary alloy the set of equations (17) and (18) can be solved analytically in the absence of convection, i.e. $U=0$. In this case modified Ivantsov functions $\mathrm{Iv}_{T}^{*}$ and $\mathrm{Iv}_{C}^{*}$ depend on the growth Péclet number $P_{g}$ only and one can write the surface concentration $C_{i}$, undercooling $\Delta T_{i}$ and $d_{0 \mathrm{CD}}$ in dependence on $P_{g}$ in a form

$$
\begin{aligned}
C_{i}\left(P_{g}\right)=\frac{C_{l \infty}}{1-\left(1-k_{0}\right) \mathrm{Iv}_{C}^{*}\left(P_{g}\right)}, \quad \Delta T_{i}\left(P_{g}\right) & =\frac{\left(1-k_{0}\right) m C_{i}\left(P_{g}\right)}{k_{0} T_{Q}}, \\
d_{0 \mathrm{CD}}\left(P_{g}\right) & =\frac{T_{Q} d_{0}}{2 m C_{i}\left(P_{g}\right)\left(1-k_{0}\right)},
\end{aligned}
$$

where

$$
\begin{array}{r}
\operatorname{Iv}_{T}^{*}\left(P_{g}\right)=P_{g} \exp \left(P_{g}\right) \int_{1}^{\infty} \frac{\exp \left(-P_{g} \eta^{\prime}\right)}{\eta^{\prime(j-1) / 2}} d \eta^{\prime}, \\
\operatorname{Iv}_{C}^{*}\left(P_{g}\right)=\frac{P_{g} D_{T}}{D_{C}} \exp \left(\frac{P_{g} D_{T}}{D_{C}}\right) \int_{1}^{\infty} \frac{\exp \left(-P_{g} D_{T} \eta^{\prime} / D_{C}\right)}{\eta^{\prime(j-1) / 2}} d \eta^{\prime} .
\end{array}
$$


Now the solvability criterion (17) reads as

$$
\begin{gathered}
\frac{d_{0}}{\sigma_{0} \alpha_{d}^{7 / n} A_{n}^{7 / n} \rho P_{g}}=\frac{1}{\left[1+a_{1} \alpha_{d}^{2 / n} A_{n}^{2 / n} P_{g}\left(1+\delta_{0} D_{T} \beta_{0} / d_{0}\right)\right]^{2}} \\
+\frac{2 k_{0} \Delta T_{i}\left(P_{g}\right) D_{T}}{\left[1+a_{2} \alpha_{d}^{2 / n} A_{n}^{2 / n} P_{g}\left(1+\delta_{0} D_{C} \beta_{0} / d_{0 \mathrm{CD}}\left(P_{g}\right)\right) D_{T} / D_{C}\right]^{2} D_{C}} \equiv \Lambda\left(P_{g}\right) .
\end{gathered}
$$

Equation (22) can be rewritten to obtain the dendrite tip diameter as a function of $P_{g}$

$$
\rho\left(P_{g}\right)=\frac{d_{0}}{\sigma_{0} \alpha_{d}^{7 / n} A_{n}^{7 / n} P_{g} \Lambda\left(P_{g}\right)} .
$$

The undercooling balance (18) is reformulated in a parametric form of $P_{g}$ as

$$
\Delta T=\Delta T_{T}\left(P_{g}\right)+\Delta T_{C}\left(P_{g}\right)+\frac{4 d_{0} T_{Q}}{\rho\left(P_{g}\right)}+\frac{2 D_{T} P_{g}}{\mu_{k} \rho\left(P_{g}\right)},
$$

where

$$
\Delta T_{T}\left(P_{g}\right)=T_{Q} \operatorname{Iv}_{T}^{*}\left(P_{g}\right), \quad \Delta T_{C}\left(P_{g}\right)=\frac{m C_{\infty}\left(1-k_{0}\right) \operatorname{Iv}_{C}^{*}\left(P_{g}\right)}{1-\left(1-k_{0}\right) \operatorname{Iv}_{C}^{*}\left(P_{g}\right)} .
$$

Concluding this section, expressions (23)-(24) represent the exact parametric solutions in the absence of convective flow.

\section{Results of dendrite growth modeling}

Comparison with data of phase field simulation. In order to obtain dendrite tip diameter and velocity, the system of equations (23)-(24) has been solved numerically with the material parameters given in Table 2 . Figure 3 demonstrates how the dendrite tip velocity $V$ depends on degree of crystal symmetry if the other material parameters remain the same. The only parameter which was varied in this calculation is the symmetry order $n$. It changes the parameter $A_{n}$ defined by equation (13). It can be seen from Figure 3 that for a given undercooling the highest symmetry order $(n=10)$ provides the largest velocity, and the lowest symmetry order $(n=2)$ gives the smallest velocity. This occurs due to the fact that the highest order of symmetry defines the fastest crystal faces with the smallest reticular atomic density. For the same order of symmetry, the dendrite tip diameter has been calculated as a function of undercooling $\Delta T$ simultaneously with the dendrite velocity $V$. Figure 4 shows that at a given undercooling the thinnest dendrite grows in case of the highest order of symmetry $(n=10)$, and the thickest dendrite grows in case of the smallest symmetry order $(n=2)$.

It is remarkable that the results of calculations by the phase field method for ice crystals with symmetry $n=2$ [4] exhibit a velocity that is higher than the dendrite velocity with the highest symmetry order $n=10$ calculated with the present sharp interface model (see Figure 3). According to the modeling results in Ref. [4], the tip diameter of ice crystals with symmetry order $n=2$ is even lower than the one for the highest order of symmetry $n=10$ calculated with the present model (see Figure 4 ). This inconsistency is explained as follows.

In phase field simulations, we could observe that the tip velocity of the ice column (symmetry $n=2$ ) [4] increases linearly with the undercooling, consistently with 


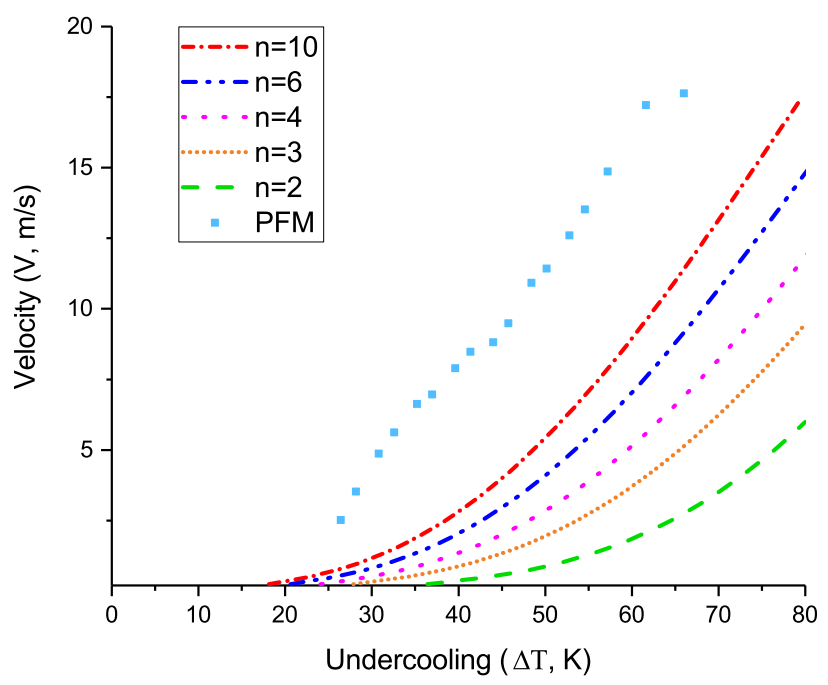

Fig. 3. Dependence of dendrite velocity on undercooling for different crystal symmetries. Points show the results of phase field modeling (PFM) of ice crystals with the lowest symmetry $(n=2)$ consistent with column shown in Figure 1(b) as described in [4].

observation [24]. This is not compatible with the analytical model for dendrite growth with symmetry $n=2$ (see green dashes in Figure 3). The explanation is that ice column growth is different in nature from dendrite growth. As opposed to dendrite growth, the selection of the ice column tip diameter and velocity are governed by attachment kinetics rather than capillary effects [25], which are therefore assumed to be negligible in the corresponding simulations. As a result, both the stability criterion (22) and the unperturbed Ivantsov's solution (21) are different for column growth and dendrite growth with symmetry $n=2[26,27]$, resulting in two different relations between tip velocity and undercooling.

Table 2. Material parameters for modeling ice dendrite growth (summarized by the calculation results from $[4,28,29])$

\begin{tabular}{llll}
\hline \hline Parameter & Symbol & Value & Units \\
\hline \hline Constant of solvability & $\sigma_{0}$ & 0.15 & - \\
Capillary constant & $d_{0}$ & $2.8 \cdot 10^{-10}$ & $\mathrm{~m}$ \\
Thermal diffusivity & $D_{T}$ & $1.2 \cdot 10^{-8}$ & $\mathrm{~m}^{2} \cdot \mathrm{s}^{-1}$ \\
Hypercooling & $T_{Q}$ & 81.56 & $\mathrm{~K}$ \\
Surface energy stiffness & $\alpha_{d}$ & 0.80 & - \\
Kinetic coefficient* & $\mu_{k}$ & 3.55 & $\mathrm{~m} /(\mathrm{s} \cdot \mathrm{K})$ \\
Stability constant & $a_{1}$ & 0.25 & - \\
Stability constant & $a_{2}$ & 0.35 & - \\
Constant & $\delta_{0}$ & 23.80 & - \\
\hline$(*) \mu_{k}$ has been calculated using the kinetic growth coefficient $\beta_{0}=0.003 \mathrm{~s} \cdot \mathrm{m}^{-1}$
\end{tabular}




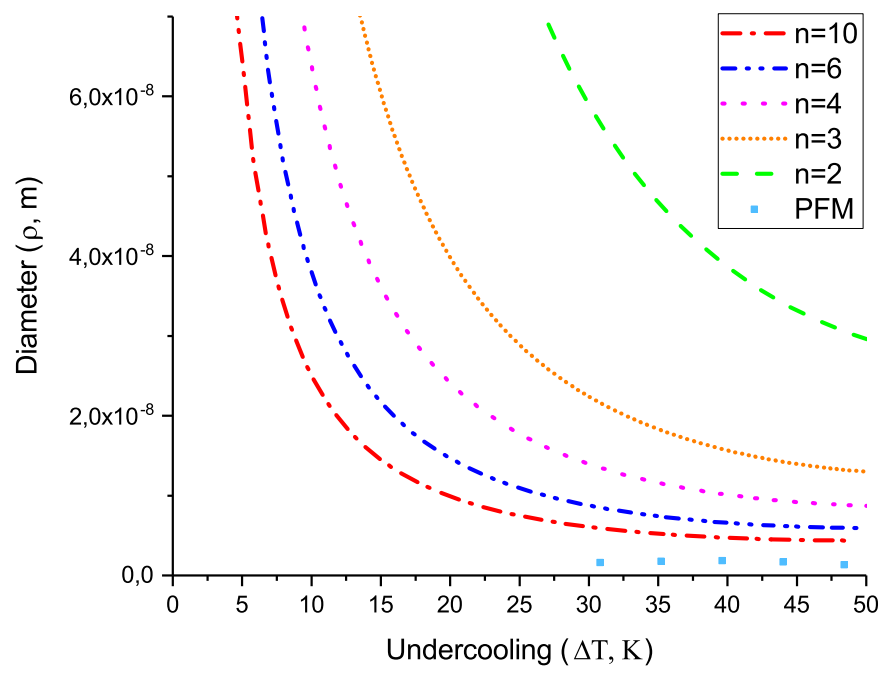

Fig. 4. Dependence of dendrite tip diameter on undercooling for different crystal symmetries. Points show the results of phase field modeling (PFM) of ice crystals with the lowest symmetry $(n=2)$ consistent with column shown in Figure 1(b) as described in [4].

Influence of convective flow. By taking into account both the n-fold symmetry and forced convection into the analytic description, allows for predictions of tip velocity and tip diameter that deviate from classical benchmark four-fold symmetry forced convection cases. For example, the six-fold symmetry of the crystalline lattice, which is encountered in ice crystals and metals such as magnesium, is shown in Figure 5. An example of numerical modeling of six-fold symmetry crystal with and without the presence of forced convection is given in this Figure. The model has an analogous statement as is given by Eqs. (1)-(9) with $C_{l}=0$, but it uses an enthalpy based approach [6] for solidification and a lattice Boltzmann method for fluid flow [8]. The model is dimensionless with the only parameter which is the anisotropy of surface energy defined by $\gamma=1-\alpha_{d} \cos (6 \theta)$. Therefore the surface stiffness which is $\gamma-d^{2} \gamma / d \theta^{2}=1-35 \alpha_{d} \cos (6 \theta)$. In the presented results the anisotropy of surface energy is $\alpha_{d}=0.5 / 35$. As a result, the crystal exhibits rotational symmetry with each arm growing in a similar fashion in the stagnant medium, Figure 5(a). When forced convection is included, in Figure 5(b) it is directed from the east to west, the incident tip grows at a faster velocity, while the downstream tip is stunted. The off-axis tips also exhibit a change in tip velocity with a slight bias to the ones incident to the flow. Such effect of convective flow has been described for crystals of four-fold symmetry using the results of phase field modeling [30-34]. It appears because the incoming flow decreases the thickness of the heat boundary layer, increases the temperature gradient and, consequently, the growth velocity of the so-called up-stream branch of a dendrite. Contrary to this, the down-stream dendrite branch has a stagnant zone in liquid which essentially decreases the crystallization heat removal. A further description and a quantitative comparison of different crystals symmetries with and without flow represent a research direction for future investigations. 

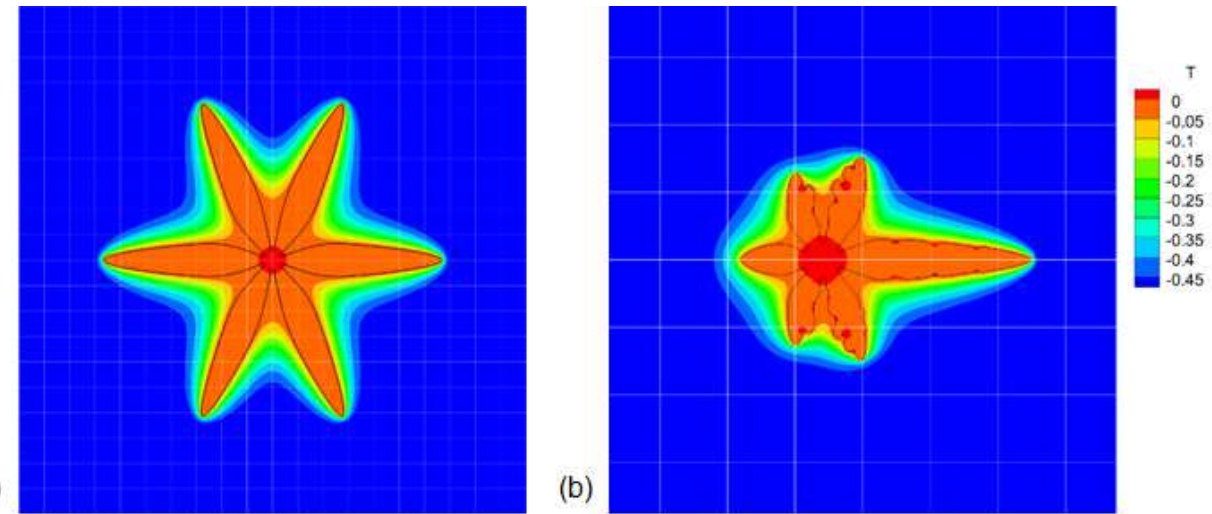

Fig. 5. Numerical simulations of a six-fold symmetry crystal with and without flow using an enthalpy based approach [6] for solidification and a lattice Boltzmann method for fluid flow [8]. (a) Stagnant fluid. (b) Forced convection directed from east to west. Numbers at the scales of colors show dimensionless temperature with respect to the adiabatic temperature of solidification (hypercooling) defined by the ratio of latent heat to specific heat.

\section{Conclusion}

A model for dendrite growth with n-fold symmetry from an undercooled liquid has been formulated. Using linear stability analysis and the solvability condition, the selection criterion for thermally and solutally controlled growth of dendrites has been found for different symmetries. Growth kinetics with different symmetry is expressed in terms of dendrite velocity $V$ and tip diameter $\rho$ as functions of undercooling $\Delta T$. It has been shown that for a given undercooling the thinnest dendrites grow fastest in case of the highest order of symmetry, and the thickest dendrites grow slowest in the case of the smallest symmetry order. These results were compared with the simulation results for growth kinetics of ice dendrites with two-fold symmetry using the phase field method. It is demonstrated that the stability criterion is different for dendrite growth and column growth as a result of different relations between tip velocity and undercooling.

\section{Acknowledgments}

L. V.T. acknowledges the support from the Ministry of Education and Science of the Russian Federation [grant number 1.12804.2018/12.2]. P. K. G. acknowledges the support by the European Space Agency (ESA) under research project MULTIPHAS (AO-2004) and the German Aerospace Center (DLR) Space Management under contract No. 50WM1541.

\section{Author contribution statement}

All authors contributed equally to the present research article.

\section{References}

1. U. Nakaya, Snow Crystals (Cambridge MA, Harvard University Press, 1954). 
2. R. Trivedi, W. Kurz, Int. Mater. Rev. 39, 49 (1994).

3. P.K. Galenko, D.V. Alexandrov, Phil. Trans. A 376, 20170210 (2018).

4. G. Demange, H. Zapolsky, R. Patte, M. Brunel, Phys. Rev. E 96, 022803 (2017).

5. G. Demange, H. Zapolsky, R. Patte, M. Brunel, Comp. Mat. 3, 15 (2017).

6. V. Voller, Int. J. Heat Mass Trans. 51, 823 (2008).

7. A. Kao, K. Pericleous, JACT 6, 173 (2011).

8. A. Kao, I. Krastins, M. Alexandrakis, N. Shevechnko, S. Eckert, K. Pericleous, JOM, 71, 48 (2019).

9. D.V. Alexandrov, P.K. Galenko, Phys. Chem. Chem. Phys. 17, 19149 (2015).

10. D.V. Alexandrov, P.K. Galenko, Phys. Rev. E 87, 062403 (2013).

11. D.V. Alexandrov, P.K Galenko, L.V. Toropova, Phil. Trans. A 376, 20170215 (2018).

12. H. Lamb (Sir), Hydrodynamics (New York, Dover Publ., 1945).

13. N.E. Kochin, I.A. Kibel, N.V. Roze, Theoretical Hydromechanics (New York, Interscience, 1964).

14. S.K. Dash, W.N. Gill, Int. J. Heat Mass Trans. 27, 1345 (1984).

15. A. Barbieri, J.S. Langer, Phys. Rev. A 39, 5314 (1989).

16. P. Pelcé, D. Bensimon, Nucl. Phys. B 2, 259 (1987).

17. P. Pelcé, Dynamics of Curved Fronts (Boston, Academic Press, 1988).

18. P. Bouissou, P. Pelcé, Phys. Rev. A 40, 6673 (1989).

19. N. Fröman, P.O. Fröman, JWKB Approximation: Contributions to the Theory (Amsterdam, North-Holland, 1965).

20. A.B. Zel'dovich, A.G. Istratov, N.I. Kidin, V.B. Librovich, Combust. Sci. Technol. 24, 1 (1980).

21. B. Caroli, C. Caroli, B. Roulet, J.S. Langer, Phys. Rev. A 33, 442 (1986).

22. D.V. Alexandrov, P.K. Galenko, Phys.-Usp. 57, 771 (2014).

23. D.V. Alexandrov, P.K. Galenko, in Proceedings of IUTAM Symposium on Recent Advances in Moving Boundary Problems in Mechanics (edited by S. Gutschmidt, J.N. Hewett, M. Sellier) (Berlin, Springer, 2019).

24. K.G. Libbrecht, J. Cryst. Growth 247, 530 (2003).

25. K.G. Libbrecht, Rep. Prog. Phys. 68, 855 (2005).

26. K.G. Libbrecht, V.M. Tanusheva, Phys. Rev. E 59, 3253 (1999).

27. K.G. Libbrecht, T. Crosby, M. Swanson, J. Cryst. Growth 240, 241 (2002).

28. D.V. Alexandrov, P.K. Galenko, J. Phys.: Condens. Matter 30, 105702 (2018).

29. D.V. Alexandrov, P.K. Galenko, J. Phys. Chem. Solids 108, 98 (2017).

30. X. Tong, C. Beckermann, A. Karma, Q. Li, Phys. Rev. E 63, 061601 (2001).

31. P.K. Galenko, D.M. Herlach, O. Funke, G. Phanikumar, in Solidification and Crystallization (Ed. D.M. Herlach) (Weinheim, Wiley-VCH, 2004).

32. J.C. Ramirez, C. Beckermann, Acta Mater. 53, 1721 (2005).

33. J-H. Jeong, N. Goldenfeld, J.A. Dantzig, Phys. Rev. E 64, 041602 (2001).

34. P.K. Galenko, D.M. Herlach, G. Phanikumar, O. Funke, in Computational Modeling and Simulation of Materials III, Part B (Edited by P. Vincenzini, A. Lami, F. Zerbetto) (Faenza, Techna Group, 2004). 